\title{
VLBA STUDIES OF BL LAC
}

\author{
G. DENN AND R. MUTEL \\ University of Iowa \\ Dept. Physics and Astronomy \\ Iowa City, IA 52240 \\ U.S.A.
}

\section{Introduction}

The radio core of BL Lac has been monitored with VLBI techniques since 1980. At least seven superluminal events have been seen, usually associated with flux outbursts. The components have been interpreted as weak relativistic shocks associated with opposed jets. Recent dramatic changes in the nucleus of BL Lac have been seen: optical spectra (Lawrence et al. 1995) show the presence of broad-line $\mathrm{H} \alpha$ and [NII] emission lines. The single-dish radio flux has also increased by 3 times in the past year. Often in BL Lac's past, a flux outburst immediately precedes the ejection of a new superluminal component.

\section{Observations}

We are currently engaged in a multifrequency monitoring program of BL Lac with the VLBA at several epochs separated by two month intervals. Table 1 lists the current status of the observations. Stokes-I maps have been produced for epochs 1995.5 and 1995.7 at all three frequencies. The $3.6 \mathrm{~cm}$ maps show one central component, slightly elongated along p.a. $=180^{\circ}$. The $2.0 \mathrm{~cm}$ and $1.3 \mathrm{~cm}$ maps show an emerging component at p.a. $=180^{\circ}$, approximately 1.25 mas from the core. This is in marked contrast to maps made as late as 1994.8 , in which the jet p.a. was nearly $200^{\circ}$. Convolving the $1.3 \mathrm{~cm}$ model with the $2.0 \mathrm{~cm}$ and $3.6 \mathrm{~cm}$ beams produced maps nearly identical to the images at those frequencies. 
TABLE 1. Observational summary (1995)

\begin{tabular}{lllll}
\hline & Jun 05 & Aug 02 & Oct 09 & Dec \\
\hline$\lambda(\mathrm{cm})$ & $\mathbf{3 . 6 , 2 . 0 , 1 . 3}$ & $\mathbf{3 . 6 , 2 . 0 , 1 . 3}$ & $2.0,1.3$ & TBD \\
Status & Correlated & Correlated & Observed & Scheduled \\
I-maps & yes & yes & not yet & - \\
P-maps & no & no & - & - \\
\hline
\end{tabular}

\section{Interpretation}

Simple conical jet models (e.g. Königl 1981) of a shocked-emission region suggests that there should be a frequency dependent separation between the core position and a shock component along the jet. For typical physical values, the ratio of distances between core and shocked component should be $D_{1.3} / D_{2}=\sim 0.75$. One-dimensional slices indicate a ratio of $D_{1.3} / D_{2}=$ $1.04 \pm 0.2$, which does not agree with the simple conical model. More realistic models (e.g. Hughes et al. ) will be tested after the polarization mapping is completed.
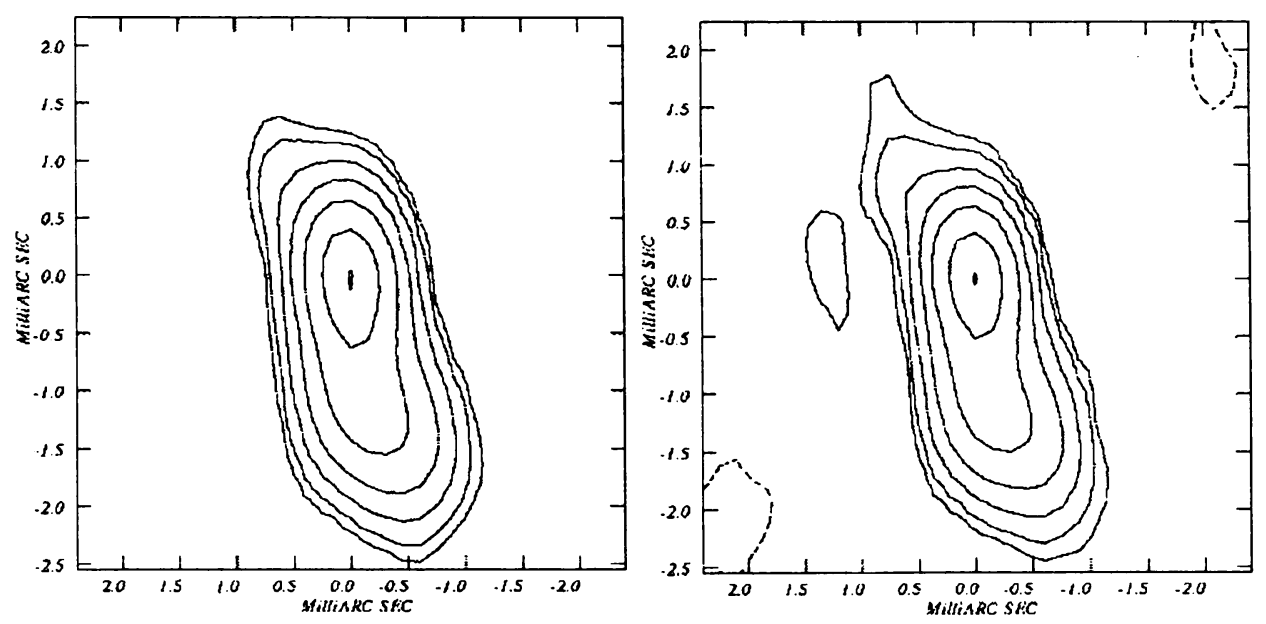

Figure 1. $15 \mathrm{GHz}$ maps of the 1995.5 and 1995.7 epochs. The lowest contour is $2 \%$.

\section{References}

Hughes, P.A., Aller, H. D., and Aller, M. F. (1989), Ap. J., 341, 68.

Königl, A., (1981), Ap. J., 243, 700.

Lawrence, et al. , (1995), preprint. 